\title{
Blutzuckerselbstkontrolle
}

\section{Wer problemorientiert misst, misst sinnvoll}

Liebe Leserinnen und Leser,

die Blutzuckerselbstkontrolle ist eines der wichtigsten Hilfsmittel in der Therapie von Menschen mit Diabetes. Sie ist das entscheidende Instrument für ein adäquates Selbstmanagement der Betroffenen. In den vergangenen Jahren ist die Zahl der Patienten, die ihren Blutzucker erfolgreich selbst messen und ihre Therapie daraufhin anpassen, kontinuierlich gestiegen.

Die Entwicklung von Blutzuckermessgeräten für die Selbstkontrolle Anfang der 1980er Jahre war eine bedeutende Innovation. Die damit verbundenen neuen Möglichkeiten einer optimierten Insulintherapie haben die Diabetologie revolutioniert und den Patienten noch mehr in den Mittelpunkt unseres therapeutischen Engagements gestellt.

Denken wir an die Anfänge zurück, war Blutzuckerselbstkontrolle ein „blutiges“ und langwieriges Verfahren. Heute können die Messgeräte Vieles, von dem wir früher nur geträumt haben. Die Minicomputer messen in kleinsten Blutmengen den Glukosegehalt, geben Trends an, interpretieren automatisch die Stoffwechselsituation, machen Vorschläge für Insulindosen und übertragen die Blutzuckerwerte in elektronische Patientenakten.

\section{Therapierelevante Technik}

Viele der neuen Gerätefunktionen erleichtern die Diabetestherapie, machen sie patientenorientierter und sicherer. So können z.B. Geräte, die Glukosewerte automatisch per Bluetooth in eine PC-Patientenakte übertragen, die Compliance und Adhärenz der Patienten stärken. Sie haben so vielleicht eher das Gefühl, dass ihre Werte zeitnah vom Arzt wahrgenommen werden. An diesem Beispiel zeigt sich wieder einmal, dass neue Technologien therapeutisch sehr relevant sein können.

\section{Diskussion zur Messung bei Typ-2-Diabetes}

Allerdings ist die Blutzuckerselbstkontrolle auch umstritten. Immer wieder wird darüber diskutiert, ob die Kosten der Maßnahme bei bestimmten Patientengruppen unter dem wachsenden Finanzdruck im Gesundheitswesen noch vertretbar sind. Damit ist die Diabetologie in der Pflicht, selbstkritisch differenzierte Vorschläge zur Indikation Selbstmessung zu erarbeiten, möglichst basierend auf der Evidenz vorliegender Studienergebnisse.

Denn die Evidenz der Blutzuckerselbstmessung ist z.T. kritisch zu betrachten, und Experten kommen zu verschiedenen Schlussfolgerungen. Der Nutzen für die Stoffwechselkontrolle unter einer intensivierten Insulintherapie bei Typ-1-Diabetes ist unstrittig und durch höchste Evidenz belegt. Das Gleiche gilt für Typ-2-Diabetiker mit dieser Therapie. Für Typ-2-Diabetiker ohne Insulinsubstitution aber ist die Datenlage widersprüchlich. Hinzu kommt die Heterogenität des Diabetesverlaufs.

\section{Die Chance, das Passende zu finden}

Wir sind als Ärzte gefordert, klare Empfehlungen für den Patienten und die Gesundheitspolitik zu etablieren. Die technischen Möglichkeiten neuer Blutzuckermessgeräte ermöglichen es, die Selbstkontrolle immer häufiger, immer einfacher und immer besser vorzunehmen. Ganz wichtig ist jedoch ein problemorientiertes und zielgerichtetes Vorgehen. Blindwütiges „Vor-sich-hin-Messen“ ist nicht sinnvoll. Es fällt in unsere ärztliche Verantwortung, die Patienten zu beraten und jenen die effektive Selbstmessung zu ermöglichen, die davon profitieren. Dann haben sie eine breite Auswahl, um das für sich passende Messgerät zu finden.

Ich wünsche Ihnen viel Spaß mit dieser Ausgabe von Info Diabetologie, die sich unter anderem mit diesem für uns in der Diabetestherapie so wichtigen Thema beschäftigt.

Peter Schwarz

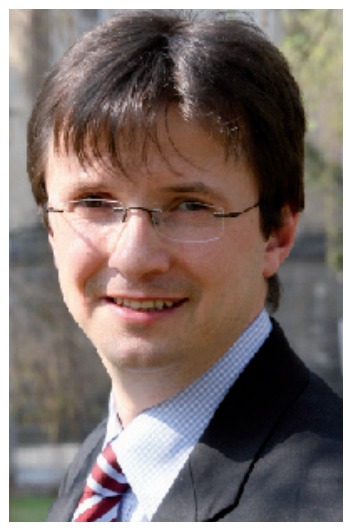

Prof. Dr. med.

Peter E. H.

Schwarz, Universitätsklinikum Carl Gustav Carus der TU Dresden, Schriftleiter Prävention Info Diabetologie 